\title{
Orbital floor fracture reduction using the prelacrimal approach: a case report
}

\author{
Geun Woo Park, MD (i), Tae Young Jung, MD $\mathbb{D}$ \\ Department of Otorhinolaryngology Head and Neck Surgery, Maryknoll Medical Center, Busan, Rep. of Korea
}

Orbital floor fractures are caused by blunt trauma to the orbital floor, the weakest part of the orbital wall. Reduction of orbital floor fractures is important to prevent complications such as diplopia and enophthalmos. The approach used in reducing orbital floor fractures can be classified into three types: transorbital, transantral, and intranasal approaches. Each approach has limitations such as scarring, numbness, and a narrow surgical view. The prelacrimal approach as a technique has the advantage of a previous intranasal approach, in that it uses the middle meatal antrostomy for the endoscopic approach and improves the limitation of the previous intranasal approach. It provides not only a wide surgical view and clear view of the fracture site, but also an easy approach to the reduction site in the outpatient department after surgery. We suggest the prelacrimal approach as a new option for the reduction of orbital floor fractures.

Keywords: endoscopy; open fracture reduction; orbital fractures

\section{Introduction}

Orbital floor fractures are caused by blunt trauma (such as car accidents, falls, violence, injuries during sports activities, industrial accidents) to the orbital wall, the weakest part of the orbital bone. This leads to herniation or incarceration of the orbital tissue. Patients with orbital floor fractures usually experience complications such as periorbital edema, diplopia, enophthalmos, pain, and facial numbness. Surgical reduction of the herniated or incarcerated orbital tissues and the fractured orbital floor is essential to prevent potential complications, such as diplopia and enophthalmos.

The existing approaches for the reduction of orbital floor fractures can be classified into transorbital, transantral, and intranasal approaches [1]. The intranasal approach uses the middle nasal meatus to reach the orbital floor. This approach involves a relatively lower risk of complications such as scarring or facial numbness compared to other approaches; however, its utility is limited by the narrow surgical view and space.

The prelacrimal approach removes maxillary sinus wall in front of the lacrimal duct to reach the orbital floor, reducing the risk of complications such as scarring. This approach also provides the advantage of a wide surgical view and space, which enables the convenient use of surgical instruments, thereby overcoming the challenges posed by the intranasal approach.

Herein, we report a successful case of orbital floor fracture reduction using the prelacrimal approach.

\section{Case report}

A 49-year-old male was hospitalized with a chief complaint of pain in the left eye and periorbital region following an impact 
injury after running into a pillar. The patient's medical history was unremarkable. Limited upward gazing and diplopia were confirmed by ophthalmic examinations conducted in the outpatient department. No enophthalmos was found on ophthalmic examination. Computed tomography confirmed left orbital floor and nasal bone fractures (Fig. 1). Since diplopia and limited ocular movement were confirmed, the patient met the criteria for reduction surgery. Reduction of the nasal bone fracture and endoscopic reduction of the orbital floor fracture using the prelacrimal approach under general anesthesia was performed.

An incision line was drawn at the anterior border of the inferior turbinate; thereafter, the nasal mucous membrane was separated from the maxillary sinus wall by a freer elevator. After separating the mucosal membrane from the maxillary sinus, the interior maxillary sinus wall was removed using an osteotome. During the process, we removed the maxillary sinus wall in front of the nasolacrimal duct to prevent any damage to the nasolacrimal duct. An orbital floor fracture inside the maxillary sinus was confirmed through the incised maxillary sinus wall. After incision of the mucosal layer of the fracture site using a sickle knife and confirmation of the fracture site border by a seeker, the fracture was reduced using an orbital maxillofacial retractor and a curved curette. After reduction, we measured the size of the fracture site using an orbital maxillofacial retractor and cut the biosorb $\beta$ plate made by OSTEONIC Co. (Seoul, Korea), which consists of poly (L)-lactide-co-glycolide and beta-tricalcium phosphate, larger than the fracture site to fully reinforce the fracture site. One cut biosorb $\beta$ plate was placed between the orbital bone and the maxillary sinus mucosal layer to support the fracture site. A fibrin glue, Tisseel (Baxter International Inc., Deerfield, IL, USA), was applied to the reduction site to fix and protect the orbital floor and plate (Fig. 2).

The diplopia and eye discomfort improved postoperatively, and computed tomography performed a month after the sur-
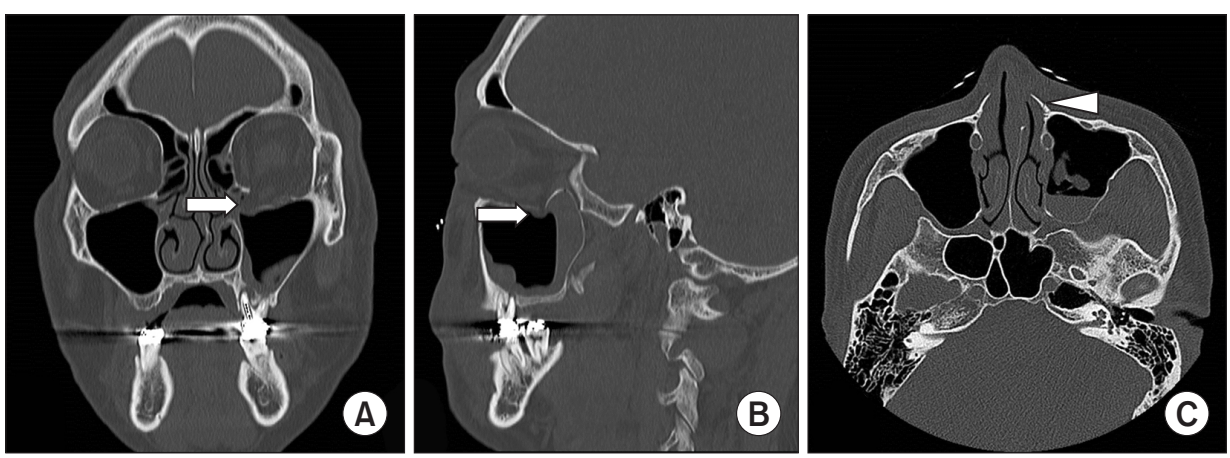

Fig. 1. Preoperative computed tomography findings showing a left inferior orbital wall fracture (arrows) (A, B) and a nasal bone fracture (arrowhead) (C).

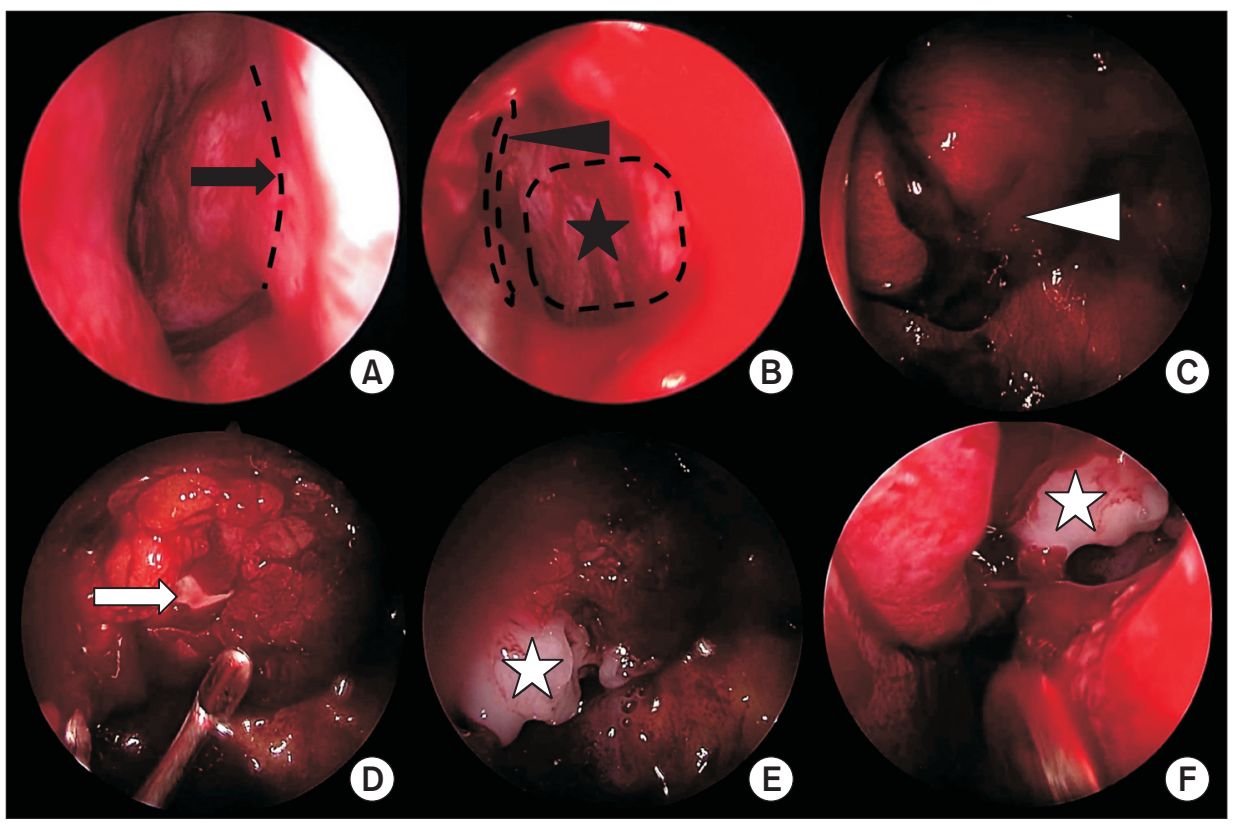

Fig. 2. Intraoperative imaging. (A) Incision line (black arrow) was drawn at the anterior border of the inferior turbinate approximately $2 \mathrm{~cm}$ vertically. (B) After separating the nasal mucous membrane from the maxillary sinus wall, interior maxillary sinus wall, approximately $3 \mathrm{~cm}^{2}$ size (black star), was removed in front of the nasolacrimal duct (black arrowhead). (C) The orbital floor was fractured and periorbital tissue was herniated into the maxillary sinus (white arrowhead). (D) Herniated periorbital tissue was reduced to the anatomic position, and a bioresorbable plate (white arrow) was placed under the fractured bone. (E, F) Fibrin glue (white stars) was sprayed to protect the reduced orbital floor and bioresorbable plate. 

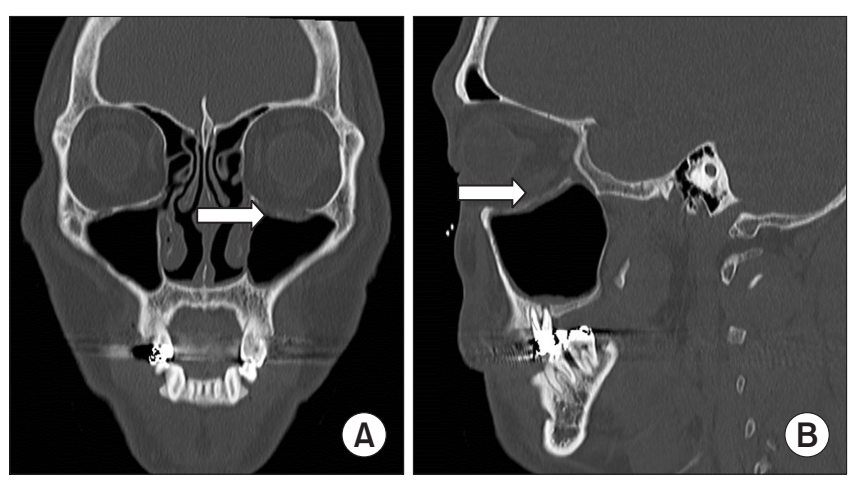

Fig. 3. Postoperative computed tomography findings showed a reduced left medial orbital wall fracture (arrows). (A) Coronal view; (B) sagittal view.

gery confirmed that the reduced orbital wall was well maintained without herniation of the periorbital tissues (Fig. 3). Currently, the patient is being monitored, and no ophthalmological abnormalities or eye discomfort have been reported up to a month after the surgery.

\section{Discussion}

Opinions on the criteria and timing of reduction surgery for orbital floor fractures vary. According to the most widely accepted Burnstine criteria, immediate surgical treatment is required if non-resolving oculocardiac reflex, white-eyed blowout fracture, or early enophthalmos is confirmed. Surgery is required within two weeks in patients with diplopia and limited ocular movement, incarcerations of the orbital tissues, or the size of the fractured area is more than $50 \%$ of the surface, with the possibility of enophthalmos confirmed by computed tomography [2].

The prelacrimal approach has been used in surgery for inverted papilloma; it provides a wider surgical field and view than the existing surgical techniques and preserves the inferior turbinate [3]. To overcome the challenges of narrow surgical vision and limited operation range posed by the existing intranasal approach, a partially modified prelacrimal approach was applied in our case. The transorbital approach uses orbital structures, such as the lower eyelid and conjunctiva, to access the orbital floor wall. It has the advantages of securing a wide surgical vision, but also involves post-surgical complications such as entropion, ectropion, and hyperproliferative scar. Due to the risk of optic nerve damage, reduction of posterior orbital floor fractures may be challenging [4]. The transantral approach uses the upper lip to enter the maxillary sinus and approach the orbital floor. This technique provides a wide surgical vision and also enables the reduction of posterior orbital floor fractures; however, postoperative complications such as facial numbness, oroantral fistula, facial edema, and recurrent sinusitis may occur $[5,6]$. The intranasal approach uses nasal endoscopy to approach the orbital floor through the middle meatus to the maxillary sinus and is associated with relatively fewer postoperative complications. However, since this approach requires tilted endoscopic and curved instruments, the surgical vision is limited, making reduction challenging, especially in cases of anterior and lateral fractures.

In comparison, the prelacrimal approach is an endoscopic approach that removes the maxillary sinus wall in front of the prelacrimal duct to approach the orbital wall through the maxillary sinus, providing sufficient surgical vision and allowing for the convenient use of instruments for fracture reduction. This is a minimally invasive approach that causes almost no postoperative complications, including scarring. It also enables accurate intraoperative localization of orbital floor fractures and convenient postoperative follow-up monitoring through the maxillary sinus. Although we proceeded under general anesthesia, depending on the patient's cooperation, this approach might be performed under monitored anesthesia care. However, complications such as active bleeding must be considered before surgery, as this approach has an invasive procedure such as an osteotome. In trapdoor fractures of the orbital floor, the best surgical outcome can be obtained using this approach. However, in extensive fractures of the orbital floor or fractures that involve the inferior orbital rim, reduction using this approach can be limited. Except for these limitation cases, this approach can be suitable for the majority of cases of orbital floor fractures.

In conclusion, we successfully performed reduction of an orbital floor fracture using the prelacrimal approach without any unusual complications, and hereby propose the prelacrimal approach as a new method for the reduction of orbital floor fractures.

\section{Conflicts of interest}

The authors have nothing to disclose.

\section{References}

1. Kwon J. Update in treatment of orbital blowout fractures. Korean J Otorhinolaryngol-Head Neck Surg 2011;54:317-23.

2. Burnstine MA. Clinical recommendations for repair of isolated orbital floor fractures: an evidence-based analysis. Ophthalmology 2002;109:1207-10; discussion 1210-1; quiz 1212-3. 
3. Suzuki M, Nakamura Y, Nakayama M, Inagaki A, Murakami $\mathrm{S}$, Takemura $\mathrm{K}$, et al. Modified transnasal endoscopic medial maxillectomy with medial shift of preserved inferior turbinate and nasolacrimal duct. Laryngoscope 2011;121:2399-401.

4. Brady SM, McMann MA, Mazzoli RA, Bushley DM, Ainbinder DJ, Carroll RB. The diagnosis and management of orbital blowout fractures: update 2001. Am J Emerg Med 2001;19:147-
54.

5. DeFreitas J, Lucente FE. The Caldwell-Luc procedure: institutional review of 670 cases: 1975-1985. Laryngoscope 1988;98:1297-300.

6. Low WK. Complications of the Caldwell-Luc operation and how to avoid them. Aust N Z J Surg 1995;65:582-4. 